\title{
Generalized Suzuki type $\alpha$-Z-contraction in b-metric space
}

\author{
Swati Antal*, U. C. Gairola
}

Department of Mathematics, H.N.B. Garhwal University, BGR Campus, Pauri Garhwal-246001, Uttarakhand, India.

\begin{abstract}
In this paper, we introduce the concept of generalized Suzuki type $\alpha$-Z-contraction concerning a simulation function $\zeta$ in b-metric space and prove the existence of fixed point results for this contraction. Our result extend the fixed point result of [A. Padcharoen, P. Kumam, P. Saipara, P. Chaipunya, Kragujevac J. Math., 42 (2018), 419-430].
\end{abstract}

Keywords: Simulation function, triangular $\alpha$-admissible mapping with respect to $\zeta$, b-metric space, generalized Suzuki type $\alpha$-Z-contraction mapping.

2010 MSC: $54 \mathrm{H} 25,47 \mathrm{H} 10$.

(C)2020 All rights reserved.

\section{Introduction and Preliminaries}

In 1993, Czerwik [4] generalized the concept of metric space by introducing a real number $s \geqslant 1$ in the triangle inequality of metric space and give the notion of b-metric spaces. Since then several papers have been published on the fixed point theory of various classes of single-valued and multi-valued operators in b-metric spaces (see, $[3,5,11,14])$.

Definition 1.1 ([4]). Let $X$ be a non-empty set and $s \geqslant 1$ be a given real number. A function $d: X \times X \rightarrow$ $[0, \infty)$ is said to be a b-metric space if, for all $x, y, z \in X$, the following conditions are satisfied:

(i) $d(x, y)=0$ iff $x=y$;

(ii) $\mathrm{d}(x, y)=\mathrm{d}(y, x)$;

(iii) $d(x, z) \leqslant s[d(x, y)+d(y, z)]$.

The pair $(X, d)$ is called a b-metric space.

It should be noted that, every metric space is a b-metric space with $s=1$ and hence the class of $\mathrm{b}$-metric spaces is larger than the class of metric spaces. But a metric space does not need to be b-metric space (see [13, example 1.4]).

\footnotetext{
${ }^{*}$ Corresponding author

Email addresses: antalswati11@gmail.com (Swati Antal), ucgairola@rediffmail .com (U. C. Gairola)

doi: $10.22436 /$ jnsa.013.04.06
}

Received: 2019-08-23 Revised: 2020-01-11 Accepted: 2020-01-16 
Definition 1.2 ([3]). Let $(X, d)$ be a b-metric space.

(i) A sequence $\left\{x_{n}\right\}$ in $X$ is called b-convergent if and only if there exists $x \in X$ such that $d\left(x_{n}, x\right) \rightarrow 0$, as $n \rightarrow \infty$. In this case, we write $\lim _{n \rightarrow \infty} x_{n} \rightarrow x$.

(ii) $\left\{x_{n}\right\}$ in $X$ is said to be b-Cauchy if and only if $d\left(x_{n}, x_{m}\right) \rightarrow 0$, as $n, m \rightarrow \infty$.

(iii) The b-metric space $(X, d)$ is said to be b-complete if every b-Cauchy sequence $\left\{x_{n}\right\}$ in $X$ is convergent.

In 2012, Samet et al. [15] introduced the concept of $\alpha$-admissible mapping.

Definition 1.3 ([15]). Let $T$ be a self mapping on $X$ and $\alpha: X \times X \rightarrow[0, \infty)$ be a function. We say that $T$ is $\alpha$-admissible, if $x, y \in X$,

$$
\alpha(x, y) \geqslant 1 \Longrightarrow \alpha(T x, T y) \geqslant 1
$$

The concept of $\alpha$-admissible mappings has been used by several researchers (see for example [1, 10]). Later, Karapinar et al. [7] introduced the notion of triangular $\alpha$-admissible mappings.

Definition 1.4 ([7]). Let $T: X \rightarrow X$ and $\alpha: X \times X \rightarrow \mathbb{R}$. Then $T$ is said to be triangular $\alpha$-admissible if

$\left(\mathrm{T}_{1}\right) \mathrm{T}$ is $\alpha$-admissible;

$\left(T_{2}\right) \alpha(x, y) \geqslant 1$ and $\alpha(y, z) \geqslant 1 \Longrightarrow \alpha(x, z) \geqslant 1, x, y, z \in X$.

Lemma 1.5 ([7]). Let $\mathrm{T}$ be a triangular $\alpha$-admissible mapping. Assume that there exists $\mathrm{x}_{0} \in \mathrm{X}$ such that $\alpha\left(x_{0}, T x_{0}\right) \geqslant 1$. Define sequence $\left\{x_{n}\right\}$ by $x_{n}=T^{n} x_{0}$. Then $\alpha\left(x_{m}, x_{n}\right) \geqslant 1$ for all $m, n \in \mathbb{N}$ with $m<n$.

Recently, in 2015, Khojasteh et al. [8] introduced the notion of simulation function with a view to consider a new class of contractions, called z-contraction with respect to a simulation function. Such family generalized the Banach contraction and unified some known nonlinear contractions.

Definition 1.6 ([8]). A simulation function is a mapping $\zeta:[0, \infty) \times[0, \infty) \rightarrow \mathbb{R}$, satisfying the following conditions:

(i) $\left(\zeta_{1}\right) \zeta(0,0)=0$;

(ii) $\left(\zeta_{2}\right) \zeta(t, s)<s-t$, for all $s, t>0$;

(iii) $\left(\zeta_{3}\right)$ if $\left\{t_{n}\right\},\left\{s_{n}\right\}$ are sequences in $(0, \infty)$ such that $\lim _{n \rightarrow \infty} t_{n}=\lim _{n \rightarrow \infty} s_{n}>0$,

then $\limsup _{n \rightarrow \infty} \zeta\left(t_{n}, s_{n}\right)<0$. We denote the set of all simulation functions by $z$.

Example $1.7([8])$. Let $\zeta:[0, \infty) \times[0, \infty) \rightarrow \mathbb{R}$, be defined by

(i) $\zeta(t, s)=\psi(s)-\phi(t)$ for all $t, s \in[0, \infty)$, where $\phi, \psi:[0, \infty) \rightarrow[0, \infty)$ are two continuous functions such that $\psi(t)=\phi(t)=0$ if and only if $t=0$ and $\psi(t)<t \leqslant \phi(t)$ for all $t>0$;

(ii) $\zeta(t, s)=s-\frac{f(t, s)}{g(t, s)} t$ for all $t, s \in[0, \infty)$, where $f, g:[0, \infty) \rightarrow[0, \infty)$ are two continuous functions with respect to each variable such that $f(t, s)>g(t, s)$ for all $t, s>0$;

(iii) $\zeta(t, s)=s-\phi(s)-t$ for all $t, s \in[0, \infty)$, where $\phi:[0, \infty) \rightarrow[0, \infty)$ is a continuous functions such that $\phi(t)=0$ if and only if $t=0$.

These are simulation functions.

Definition $1.8([8])$. Let $(X, d)$ be a metric space, $T: X \rightarrow X$ be a mapping and $\zeta \in Z$. Then $T$ is called a $z$-contraction with respect to $\zeta$ if the following condition is satisfied

$$
\zeta(d(T x, T y), d(x, y)) \geqslant 0,
$$

for all $x, y \in X$. 
Later, in 2017, Kumam et al. [9] introduce the notion of Suzuki type Z-contraction as follows.

Definition 1.9 ([9]). Let $(X, d)$ be a metric space, $T: X \rightarrow X$ be a mapping and $\zeta \in Z$. Then $T$ is called a Suzuki type $z$-contraction with respect to $\zeta$, if the following condition is satisfied

$$
\frac{1}{2} d(x, T x)<d(x, y) \Rightarrow \zeta(d(T x, T y), d(x, y)) \geqslant 0
$$

for all $x, y \in X$, with $x \neq y$.

Remark 1.10 ([9]). It is clear from the definition of simulation function that $\zeta(t, s)<s-t \leqslant 0$, for all $t \geqslant s>0$. Therefore if $T$ is a Suzuki type Z-contraction with respect to $\zeta$, then

$$
\frac{1}{2} d(x, T x)<d(x, y) \Rightarrow d(T x, T y)<d(x, y),
$$

for all distinct $x, y \in X$.

Theorem 1.11 ([9]). Let $(\mathrm{X}, \mathrm{d})$ be a metric space and $\mathrm{T}: \mathrm{X} \rightarrow \mathrm{X}$ be a Suzuki type Z-contraction with respect to $\zeta \in Z$. Then $T$ has at most one fixed point.

In 2018, Padcharoen et al. [12] introduced the generalized Suzuki type Z-contraction in metric space as follows.

Definition 1.12 ([12]). Let $(X, d)$ be a metric space, $T: X \rightarrow X$ a mapping and $\zeta \in Z$. Then $T$ is called a generalized Suzuki type $z$-contraction with respect to $\zeta$, if the following condition is satisfied

$$
\frac{1}{2} d(x, T x)<d(x, y) \Rightarrow \zeta(d(T x, T y), M(x, y)) \geqslant 0,
$$

for all distinct $x, y \in X$, where

$$
M(x, y)=\max \left\{d(x, y), d(x, T x), d(y, T y), \frac{d(x, T y)+d(y, T x)}{2}\right\} .
$$

Motivated and inspired by Definition 1.12 and the work of Babu et al. [2], we introduced the definition of generalized Suzuki type $\alpha$-z-contraction with respect to $\zeta$ in $b$-metric space.

Definition 1.13. Let $(X, d)$ be a b-metric space with coefficient $s \geqslant 1$ and $\alpha: X \times X \rightarrow \mathbb{R}$ be a function. A mapping $T: X \rightarrow X$ is said to be a generalized Suzuki type $\alpha-Z$ contraction with respect to $\zeta$ if there exists a simulation function $\zeta \in Z$ such that

$$
\frac{1}{2 s} d(x, T x)<d(x, y) \Rightarrow \zeta\left(s^{4} \alpha(x, y) d(T x, T y), M_{T}(x, y)\right) \geqslant 0,
$$

for all distinct $x, y \in X$, where

$$
M_{T}(x, y)=\max \left\{d(x, y), d(x, T x), d(y, T y), \frac{d(x, T y)+d(y, T x)}{2 s}\right\} .
$$

Remark 1.14. It is clear from the definition of simulation function that $\zeta(t, s)<s-t \leqslant 0$, for all $t \geqslant s>0$. Therefore if $\mathrm{T}$ is a generalized Suzuki type $\alpha$ - $Z$-contraction with respect to $\zeta$, then

$$
\left.\frac{1}{2 s} d(x, T x)<d(x, y) \Rightarrow s^{4} \alpha(x, y) d(T x, T y)<M(x, y)\right)
$$

for all distinct $x, y \in X$.

\section{Main result}

Theorem 2.1. Let $(X, d)$ be a complete b-metric space with coefficient $s \geqslant 1$ and $\alpha: X \times X \rightarrow \mathbb{R}$ be a function. Let $\mathrm{T}: \mathrm{X} \rightarrow \mathrm{X}$ be a self mapping and $\zeta \in Z$. Suppose that the following conditions are satisfied:

(i) $T$ is generalized Suzuki type $\alpha$-Z-contraction with respect to $\zeta$; 
(ii) $\mathrm{T}$ is a triangular $\alpha$-admissible;

(iii) there exists $\mathrm{x}_{0} \in \mathrm{X}$ such that $\alpha\left(\mathrm{x}_{0}, \mathrm{~T} \mathrm{x}_{0}\right) \geqslant 1$;

(iv) either $T$ is continuous or for any sequence $\left\{x_{n}\right\}$ in $X$ with $\alpha\left(x_{n}, x_{n+1}\right) \geqslant 1$ for all $n \in \mathbb{N}_{0}$ such that $\mathrm{x}_{\mathrm{n}} \rightarrow \mathrm{x} \in \mathrm{X}$ as $\mathrm{n} \rightarrow \infty$, we have $\alpha\left(\mathrm{x}_{\mathrm{n}}, \mathrm{x}\right) \geqslant 1$ for all $\mathrm{n} \in \mathbb{N}_{0}$.

Then $\mathrm{T}$ has a fixed point $\mathrm{x}^{*} \in \mathrm{X}$.

Proof. By assumption (iii), there exists a point $x_{0} \in X$ such that $\alpha\left(x_{0}, T x_{0}\right) \geqslant 1$. Define a sequence $\left\{x_{n}\right\}$ in $X$ by $x_{n+1}=T x_{n}$ for all $n \in \mathbb{N}_{0}$ (where $\mathbb{N}_{0}=\mathbb{N} \cup\{0\}, \mathbb{N}=$ set of natural numbers). If there exists an $n_{0}$ such that $x_{n_{0}+1}=x_{n_{0}}$ for some $n_{0} \in \mathbb{N}_{0}$, then $x_{n_{0}}$ is a fixed point of $T$, which completes the proof. Therefore we assume that $x_{n} \neq x_{n+1}$, for all $n \in \mathbb{N}_{0}$. Hence we have

$$
\frac{1}{2 s} d\left(x_{n}, T x_{n}\right)<d\left(x_{n}, x_{n+1}\right) \text { for all } n \in \mathbb{N}_{0} .
$$

The mapping $\mathrm{T}$ is triangular $\alpha$-admissible by Lemma 1.5 , we have

$$
\alpha\left(x_{n}, x_{n+1}\right) \geqslant 1 \text {, for all } n \in \mathbb{N}_{0} .
$$

Then by (1.1), we have

$$
0 \leqslant \zeta\left(s^{4} \alpha\left(x_{n}, x_{n+1}\right) d\left(T x_{n}, T x_{n+1}\right), M_{T}\left(x_{n}, x_{n+1}\right)<M_{T}\left(x_{n}, x_{n+1}\right)-s^{4} \alpha\left(x_{n}, x_{n+1}\right) d\left(T x_{n}, T x_{n+1}\right) .\right.
$$

Consequently, we drive that

$$
d\left(x_{n+1}, x_{n+2}\right) \leqslant s^{4} \alpha\left(x_{n}, x_{n+1}\right) d\left(T x_{n}, T x_{n+1}\right)<M_{T}\left(x_{n}, x_{n+1}\right) .
$$

Thus we have

$$
d\left(x_{n+1}, x_{n+2}\right)<M_{T}\left(x_{n}, x_{n+1}\right),
$$

where

$$
\begin{aligned}
M_{T}\left(x_{n}, x_{n+1}\right) & =\max \left\{d\left(x_{n}, x_{n+1}\right), d\left(x_{n}, T x_{n}\right), d\left(x_{n+1}, T x_{n+1}\right), \frac{d\left(x_{n}, T x_{n+1}\right)+d\left(x_{n+1}, T x_{n}\right)}{2 s}\right\} \\
& =\max \left\{d\left(x_{n}, x_{n+1}\right), d\left(x_{n}, x_{n+1}\right), d\left(x_{n+1}, x_{n+2}\right), \frac{d\left(x_{n}, x_{n+2}\right)+d\left(x_{n+1}, x_{n+1}\right)}{2 s}\right\} \\
& =\max \left\{d\left(x_{n}, x_{n+1}\right), d\left(x_{n+1}, x_{n+2}\right), \frac{d\left(x_{n}, x_{n+2}\right)}{2 s}\right\} .
\end{aligned}
$$

Since

$$
\frac{d\left(x_{n}, x_{n+2}\right)}{2 s} \leqslant \frac{s\left[d\left(x_{n}, x_{n+1}\right)+d\left(x_{n+1}, x_{n+2}\right)\right]}{2 s} \leqslant \max \left\{d\left(x_{n}, x_{n+1}\right), d\left(x_{n+1}, x_{n+2}\right)\right\},
$$

then we get

$$
M_{T}\left(x_{n}, x_{n+1}\right) \leqslant \max \left\{d\left(x_{n}, x_{n+1}\right), d\left(x_{n+1}, x_{n+2}\right)\right\}
$$

If $d\left(x_{n}, x_{n+1}\right)<d\left(x_{n+1}, x_{n+2}\right)$, then

$$
\max \left\{d\left(x_{n}, x_{n+1}\right), d\left(x_{n+1}, x_{n+2}\right)\right\}=d\left(x_{n+1}, x_{n+2}\right) .
$$

Then (2.1) becomes

$$
d\left(x_{n+1}, x_{n+2}\right)<d\left(x_{n+1}, x_{n+2}\right),
$$


which is a contradiction. Thus we conclude that

$$
d\left(x_{n+1}, x_{n+2}\right)<d\left(x_{n}, x_{n+1}\right) .
$$

Which implies that $d\left(x_{n}, x_{n+1}\right)$ is monotonically decreasing sequence of non negative real numbers. Thus there exists $r \geqslant 0$, such that $\lim _{n \rightarrow \infty} d\left(x_{n}, x_{n+1}\right)=r$. We shall prove that $r=0$. Suppose on the contrary that $r>0$. Letting $t_{n}=\alpha\left(x_{n}, x_{n+1}\right) d\left(x_{n+1}, x_{n+2}\right)$ and $s_{n}=d\left(x_{n}, x_{n+1}\right)$ and using $\left(\zeta_{3}\right)$, we get

$$
0 \leqslant \limsup _{n \rightarrow \infty} \zeta\left(s^{4} \alpha\left(x_{n}, x_{n+1}\right) d\left(x_{n+1}, x_{n+2}\right), d\left(x_{n}, x_{n+1}\right)\right)<0,
$$

which is a contradiction. Thus we conclude that $r=0$, i.e.,

$$
\lim _{n \rightarrow \infty} d\left(x_{n}, x_{n+1}\right)=0 .
$$

Now, we shall prove that $\left\{x_{n}\right\}$ is a Cauchy sequence. Suppose on the contrary that $\left\{x_{n}\right\}$ is not a Cauchy sequence. Thus there exist $\epsilon>0$ and the sequences $\{u(n)\}_{\mathfrak{n}=1}^{\infty}$ and $\{v(n)\}_{\mathfrak{n}=1}^{\infty}$ of natural numbers such that

$$
u(n)>v(n)>n, d\left(x_{u(n)}, x_{v(n)}\right) \geqslant \epsilon .
$$

Moreover, corresponding to $v(n)$, we can choose the smallest $u(n)$ satisfying (2.4). Then

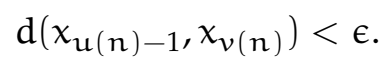

By using (2.4), (2.5), and the triangle inequality, we get

$$
\epsilon \leqslant d\left(x_{\mathfrak{u}(n)}, x_{v(n)}\right) \leqslant s\left[d\left(x_{\mathfrak{u}(n)}, x_{\mathfrak{u}(n)-1}\right)+d\left(x_{\mathfrak{u}(n)-1}, x_{v(n)}\right)\right] \leqslant s d\left(x_{\mathfrak{u}(n)}, x_{\mathfrak{u}(n)-1}\right)+s \epsilon .
$$

Taking the upper and lower limits as $n \rightarrow \infty$ and using (2.3), we get

$$
\epsilon \leqslant \liminf _{n \rightarrow \infty} d\left(x_{u(n)}, x_{v(n)}\right) \leqslant \limsup _{n \rightarrow \infty} d\left(x_{u(n)}, x_{v(n)}\right) \leqslant s \epsilon .
$$

Again by the triangle inequality, we have

$$
\epsilon \leqslant d\left(x_{\mathfrak{u}(n)}, x_{v(n)}\right) \leqslant s\left[d\left(x_{\mathfrak{u}(n)}, x_{v(n)+1}\right)+d\left(x_{v(n)+1}, x_{v(n)}\right)\right]
$$

and

$$
d\left(x_{\mathfrak{u}(n)}, x_{v(n)+1}\right) \leqslant s\left[d\left(x_{\mathfrak{u}(n)}, x_{v(n)}\right)+d\left(x_{v(n)}, x_{v(n)+1}\right)\right] .
$$

So from (2.3), (2.6), (2.7), and (2.8), we have

$$
\frac{\epsilon}{s} \leqslant \limsup _{n \rightarrow \infty} d\left(x_{\mathfrak{u}(n)}, x_{v(n)+1}\right) \leqslant s^{2} \epsilon .
$$

Again, using above process we get

$$
\frac{\epsilon}{s} \leqslant \limsup _{n \rightarrow \infty} d\left(x_{u(n)+1}, x_{v(n)}\right) \leqslant s^{2} \epsilon .
$$

By the triangle inequality

$$
d\left(x_{\mathfrak{u}(n)}, x_{v(n)+1}\right) \leqslant s\left[d\left(x_{\mathfrak{u}(n)}, x_{\mathfrak{u}(n)+1}\right)+d\left(x_{\mathfrak{u}(n)+1}, x_{v(n)+1}\right)\right] .
$$

Now using (2.3) and (2.9)

$$
\frac{\epsilon}{s^{2}} \leqslant \limsup _{n \rightarrow \infty} d\left(x_{\mathfrak{u}(n)+1}, x_{v(n)+1}\right)
$$


By the triangle inequality

$$
\begin{aligned}
d\left(x_{\mathfrak{u}(n)+1}, x_{v(n)+1}\right) & \leqslant s\left[d\left(x_{\mathfrak{u}(n)+1}, x_{v(n)}\right)+d\left(x_{v(n)}, x_{v(n)+1}\right)\right] \\
& \leqslant s^{2}\left[d\left(x_{\mathfrak{u}(n)+1}, x_{\mathfrak{u}(n)}\right)+d\left(x_{\mathfrak{u}(n)}, x_{v(n)}\right)\right]+s d\left(x_{v(n)}, x_{v(n)+1}\right) .
\end{aligned}
$$

Using (2.6)

$$
\limsup _{n \rightarrow \infty} d\left(x_{\mathfrak{u}(n)+1}, x_{v(n)+1}\right) \leqslant s^{3} \epsilon
$$

So from (2.11) and (2.12), we have

$$
\frac{\epsilon}{s^{2}} \leqslant \limsup _{n \rightarrow \infty} d\left(x_{\mathfrak{u}(n)+1}, x_{v(n)+1}\right) \leqslant s^{3} \epsilon
$$

Similarly, we can obtain

$$
\frac{\epsilon}{s^{2}} \leqslant \liminf _{n \rightarrow \infty} d\left(x_{\mathfrak{u}(n)+1}, x_{v(n)+1}\right) \leqslant s^{3} \epsilon
$$

Using (2.13) and (2.14), we have

$$
\frac{\epsilon}{s^{2}} \leqslant \liminf _{n \rightarrow \infty} d\left(x_{\mathfrak{u}(n)+1}, x_{v(n)+1}\right) \leqslant \limsup _{n \rightarrow \infty} d\left(x_{\mathfrak{u}(n)+1}, x_{v(n)+1}\right) \leqslant s^{3} \epsilon .
$$

Now from (2.3), (2.4), and (2.5), we can choose a positive integer $n_{1} \in \mathbb{N}$ such that

$$
\frac{1}{2 s} d\left(x_{\mathfrak{u}(n)}, T x_{\mathfrak{u}(n)}\right)<\frac{\epsilon}{2 s}<d\left(x_{\mathfrak{u}(n)}, x_{v(n)}\right), \quad \forall n \geqslant n_{1} .
$$

Then by assumption of the theorem for every $n \geqslant n_{1}$ and by Lemma 1.5, we have $\alpha\left(x_{\mathfrak{u}(n)}, x_{v(n)}\right) \geqslant 1$. Then from (1.1), we have

$$
\begin{aligned}
0 & \leqslant \zeta\left(s^{4} \alpha\left(x_{\mathfrak{u}(n)}, x_{v(n)}\right) d\left(x_{\mathfrak{u}(n)+1}, x_{v(n)+1}\right), M_{T}\left(x_{\mathfrak{u}(n)}, x_{v(n)}\right)\right) \\
& <M_{T}\left(x_{\mathfrak{u}(n)}, x_{v(n)}\right)-s^{4} \alpha\left(x_{\mathfrak{u}(n)}, x_{v(n)}\right) d\left(x_{\mathfrak{u}(n)+1}, x_{v(n)+1}\right),
\end{aligned}
$$

which is equivalent to

$$
d\left(x_{\mathfrak{u}(n)+1}, x_{v(n)+1}\right) \leqslant s^{4} \alpha\left(x_{\mathfrak{u}(n)}, x_{v(n)}\right) d\left(x_{\mathfrak{u}(n)+1}, x_{v(n)+1}\right)<M_{T}\left(x_{\mathfrak{u}(n)}, x_{v(n)}\right),
$$

where

$$
\begin{aligned}
M_{T}\left(x_{\mathfrak{u}(n)}, x_{v(n)}\right)= & \max \left\{d\left(x_{\mathfrak{u}(n)}, x_{v(n)}\right), d\left(x_{\mathfrak{u}(n)}, T x_{\mathfrak{u}(n)}\right), d\left(x_{v(n)}, T x_{v(n)}\right),\right. \\
& \left.\frac{d\left(x_{\mathfrak{u}(n)}, T x_{v(n)}\right)+d\left(x_{v(n)}, T x_{\mathfrak{u}(n)}\right)}{2 s}\right\} \\
= & \max \left\{d\left(x_{\mathfrak{u}(n)}, x_{v(n)}\right), d\left(x_{\mathfrak{u}(n)}, x_{\mathfrak{u}(n)+1}\right), d\left(x_{v(n)}, x_{v(n)+1}\right),\right. \\
& \left.\frac{d\left(x_{\mathfrak{u}(n)}, x_{v(n)+1}\right)+d\left(x_{v(n)}, x_{\mathfrak{u}(n)+1}\right)}{2 s}\right\} .
\end{aligned}
$$

Taking the upper limit as $n \rightarrow \infty$ on each side of the above inequality and using (2.6), (2.9), and (2.10), we have

$$
\limsup _{n \rightarrow \infty} M_{T}\left(x_{\mathfrak{u}(n)}, x_{v(n)}\right)=\limsup _{n \rightarrow \infty}\left[\max \left\{s \epsilon, 0,0, \frac{s^{2} \epsilon+s^{2} \epsilon}{2 s}\right\}\right]=s \epsilon
$$


Therefore from (2.16) taking upper limit and using (2.15), we get

$$
\begin{aligned}
0 & \leqslant \limsup _{n \rightarrow \infty} \zeta\left(s^{4} \alpha\left(x_{\mathfrak{u}(n)}, x_{v(n)}\right) d\left(x_{\mathfrak{u}(n)+1}, x_{v(n)+1}\right), M_{T}\left(x_{\mathfrak{u}(n)}, x_{v(n)}\right)\right) \\
& <\limsup _{n \rightarrow \infty}\left[M_{T}\left(x_{\mathfrak{u}(n)}, x_{v(n)}\right)-s^{4} \alpha\left(x_{\mathfrak{u}(n)}, x_{v(n)}\right) d\left(x_{\mathfrak{u}(n)+1}, x_{v(n)+1}\right)\right] \\
& \leqslant \limsup _{n \rightarrow \infty} M_{T}\left(x_{\mathfrak{u}(n)}, x_{v(n)}\right)-s^{4} \alpha\left(x_{\mathfrak{u}(n)}, x_{v(n)}\right) \liminf _{n \rightarrow \infty} d\left(x_{\mathfrak{u}(n)+1}, x_{v(n)+1}\right) \\
& \leqslant s \epsilon-s^{4} \alpha\left(x_{\mathfrak{u}(n)}, x_{v(n)}\right)\left(\frac{\epsilon}{s^{2}}\right)<0,
\end{aligned}
$$

which is a contradiction. Hence $\left\{x_{n}\right\}$ is a Cauchy sequence in $(X, d)$. Since $X$ is complete b-metric space, then there exists $x^{*} \in X$ such that

$$
\lim _{n \rightarrow \infty} x_{n}=x^{*}
$$

Now, we show that $x^{*}$ is a fixed point of T. Assume that (iv) holds, then $\alpha\left(x_{n}, x^{*}\right) \geqslant 1$. We claim that, for every $n \in \mathbb{N}$,

$$
\frac{1}{2 s} d\left(x_{n}, T x_{n}\right)<d\left(x_{n}, x^{*}\right) \text { or } \frac{1}{2 s} d\left(T x_{n}, T^{2} x_{n}\right)<d\left(T x_{n}, x^{*}\right) .
$$

Suppose on the contrary that there exists $m \in \mathbb{N}$, such that

$$
\frac{1}{2 s} d\left(x_{m}, T x_{m}\right) \geqslant d\left(x_{m}, x^{*}\right) \text { and } \frac{1}{2 s} d\left(T x_{m}, T^{2} x_{m}\right) \geqslant d\left(T x_{m}, x^{*}\right) .
$$

Therefore

$$
2 s d\left(x_{m}, x^{*}\right) \leqslant d\left(x_{m}, T x_{m}\right) \leqslant s\left[d\left(x_{m}, x^{*}\right)+d\left(x^{*}, T x_{m}\right)\right] .
$$

Which implies that

$$
d\left(x_{m}, x^{*}\right) \leqslant d\left(x^{*}, T x_{m}\right) .
$$

Now, from (2.2) and (2.20) we have

$$
d\left(T x_{m}, T^{2} x_{m}\right)<d\left(x_{m}, T x_{m}\right) \leqslant s\left[d\left(x_{m}, x^{*}\right)+d\left(x^{*}, T x_{m}\right)\right] \leqslant 2 s d\left(x^{*}, T x_{m}\right) .
$$

It follows from (2.19) and (2.21) that

$$
d\left(T x_{m}, T^{2} x_{m}\right)<d\left(T x_{m}, T^{2} x_{m}\right) .
$$

This is a contradiction. Hence (2.18) holds. If part (i) of (2.18) is true, by generalized Suzuki type $\alpha$ $z$-contraction with respect to $\zeta$, we have

$$
0 \leqslant \zeta\left(s^{4} \alpha\left(x_{n}, x^{*}\right) d\left(T x_{n}, T x^{*}\right), M_{T}\left(x_{n}, x^{*}\right)\right)<M_{T}\left(x_{n}, x^{*}\right)-s^{4} \alpha\left(x_{n}, x^{*}\right) d\left(T x_{n}, T x^{*}\right),
$$

which is equivalent to

$$
d\left(T x_{n}, T x^{*}\right) \leqslant s^{4} \alpha\left(x_{n}, x^{*}\right) d\left(T x_{n}, T x^{*}\right)<M_{T}\left(x_{n}, x^{*}\right),
$$

where

$$
M_{T}\left(x_{n}, x^{*}\right)=\max \left\{d\left(x_{n}, x^{*}\right), d\left(x_{n}, T x_{n}\right), d\left(x^{*}, T x^{*}\right), \frac{d\left(x_{n}, T x^{*}\right)+d\left(x^{*}, T x_{n}\right)}{2 s}\right\} .
$$


Letting $n \rightarrow \infty$ and by using (2.17), we obtain

$$
\lim _{n \rightarrow \infty} M_{T}\left(x_{n}, x^{*}\right)=d\left(x^{*}, T x^{*}\right) .
$$

By using (2.21), (2.22), (iv), and $\left(\zeta_{3}\right)$, we have

$$
\begin{aligned}
0 & \leqslant \zeta\left(s^{4} \alpha\left(x_{n}, x^{*}\right) d\left(T x_{n}, T x^{*}\right), M_{T}\left(x_{n}, x^{*}\right)\right) \\
& \leqslant \limsup _{n \rightarrow \infty} \zeta\left(s^{4} \alpha\left(x_{n}, x^{*}\right) d\left(T x_{n}, T x^{*}\right), M_{T}\left(x_{n}, x^{*}\right)\right) \\
& \leqslant \limsup _{n \rightarrow \infty}\left[M_{T}\left(x_{n}, x^{*}\right)-s^{4} \alpha\left(x_{n}, x^{*}\right) d\left(T x_{n}, T x^{*}\right)\right] .
\end{aligned}
$$

According to property $\left(\zeta_{3}\right)$ from Definition 1.6, since the both sequences $d\left(T x_{n}, T x^{*}\right), M_{T}\left(x_{n}, x^{*}\right)$ converge to the $d\left(x^{*}, T x^{*}\right)>0$. By assumption it is clear that

$$
0 \leqslant \limsup _{n \rightarrow \infty} \zeta\left(s^{4} \alpha\left(x_{n}, x^{*}\right) d\left(T x_{n}, T x^{*}\right), M_{T}\left(x_{n}, x^{*}\right)\right)<0,
$$

which is a contradiction. Hence $x^{*}=T x^{*}$, i.e., $x^{*}$ is a fixed point of T. If part (ii) of (2.18) is true, using a similar method to the above, we get $x^{*}=T x^{*}$. Hence $x^{*}$ is a fixed point of $T$.

Now, we prove the uniqueness of the fixed point result. We need the following additional condition.

(A) For all $x^{*}, y^{*} \in \operatorname{Fix}(T)$, there exists $z \in X$ such that $\alpha\left(x^{*}, z\right) \geqslant 1$ and $\alpha\left(y^{*}, z\right) \geqslant 1$, where $\operatorname{Fix}(T)$ denotes the set of fixed points of $\mathrm{T}$.

Theorem 2.2. By adding condition (A) to the hypothesis of Theorem 2.1, we obtain that $x^{*}$ is the unique fixed point of $T$.

Proof. We argue by contradiction, i.e., if $x^{*}, y^{*} \in X$ are two fixed points of $T$, such that $x^{*} \neq y^{*}$. Since $T$ is triangular $\alpha$-admissible and by assumption (A), we have $\alpha\left(x^{*}, y^{*}\right) \geqslant 1$, then we have $0=\frac{1}{2 s} d\left(x^{*}, T x^{*}\right)<$ $d\left(x^{*}, y^{*}\right)$ and from (1.1), we obtain

$$
\zeta\left(s^{4} \alpha\left(x^{*}, y^{*}\right) d\left(T x^{*}, T y^{*}\right), M_{T}\left(x^{*}, y^{*}\right)\right) \geqslant 0,
$$

where

$$
M_{T}\left(x^{*}, y^{*}\right)=\max \left\{d\left(x^{*}, y^{*}\right), d\left(x^{*}, T x^{*}\right), d\left(y^{*}, T y^{*}\right), \frac{d\left(x^{*}, T y^{*}\right)+d\left(y^{*}, T x^{*}\right)}{2 s}\right\}=d\left(x^{*}, y^{*}\right) .
$$

So, by (2.23), we have

$$
\begin{aligned}
0 \leqslant \zeta\left(s^{4} \alpha\left(x^{*}, y^{*}\right) d\left(T x^{*}, T y^{*}\right), M_{T}\left(x^{*}, y^{*}\right)\right) & =\zeta\left(s^{4} \alpha\left(x^{*}, y^{*}\right) d\left(x^{*}, y^{*}\right), d\left(x^{*}, y^{*}\right)\right) \\
& <d\left(x^{*}, y^{*}\right)-s^{4} \alpha\left(x^{*}, y^{*}\right) d\left(x^{*}, y^{*}\right) \leqslant 0
\end{aligned}
$$

which is a contradiction. Hence, $x^{*}=y^{*}$.

Example 2.3. Let $X=\{1,2,3,4,5\}$ and $d: X \times X \rightarrow[0, \infty)$ be defined as follows: $d(1,2)=d(2,4)=d(3,5)=$ $1, \mathrm{~d}(1,5)=1.02, \mathrm{~d}(1,3)=\mathrm{d}(3,4)=1.5, \mathrm{~d}(1,4)=\mathrm{d}(2,5)=\mathrm{d}(4,5)=2.4, \mathrm{~d}(2,3)=3, \mathrm{~d}(1,1)=\mathrm{d}(2,2)=$ $d(3,3)=d(4,4)=d(5,5)=0$, and $d(x, y)=d(y, x)$ for all $x, y \in X$. As $3=d(2,3) \not \leq d(2,1)+d(1,3)=2.5$, $d$ is not a metric on $X$. Clearly $(X, d)$ is a complete b-metric space with parameter $s=\frac{6}{5}$. We define $\mathrm{T}: \mathrm{X} \rightarrow \mathrm{X}$ such that

$$
\mathrm{T}(1)=\mathrm{T}(2)=\mathrm{T}(5)=2, \mathrm{~T}(3)=5, \text { and } \mathrm{T}(4)=1 .
$$

Let $A=\{(1,1),(1,2),(2,1),(2,2),(2,5),(5,2),(5,5),(1,5),(5,1),(3,4),(4,3),(3,3),(4,4)\}$, and $\alpha: X \times X \rightarrow$ $\mathbb{R}$ by 


$$
\alpha(x, y)=\left\{\begin{array}{lc}
1, & \text { if }(x, y) \in A, \\
0, & \text { otherwise }
\end{array}\right.
$$

Let $\zeta:[0, \infty) \times[0, \infty) \rightarrow \mathbb{R}$ defined by $\zeta(t, s)=\frac{11}{12} s-t$ for all $s, t \in[0, \infty)$. Now we show that $\mathrm{T}$ is $\alpha$-admissible. If $x, y \in\{1,2,5\}$, then $\alpha(x, y)=1$ implies that $\alpha(T x, T y)=\alpha(2,2)=1$. If $x, y \in\{3,4\}$ then, $\alpha(3,4)=1$ implies that $\alpha(\mathrm{T} 3, \mathrm{~T} 4)=\alpha(5,1)=1$. Thus for any $x, y \in X, \alpha(x, y)=1$ implies that $\alpha(T x, T y)=1$. Therefore $T$ is $\alpha$-admissible. If $x, y, z \in\{1,2,5\}$, then $\alpha(x, y)=1$ and $\alpha(y, z)=1$ implies that $\alpha(x, z)=1$. If $x, y \in\{3,4\}$, then $\alpha(x, z)=1$ and $\alpha(y, z)=1$ implies that $\alpha(x, y)=1$. Thus for any $x, y, z \in X, \alpha(x, z)=1$ and $\alpha(z, y)=1$ implies that $\alpha(x, y)=1$. Therefore $T$ is triangular $\alpha$-admissible mapping. Now we verify the inequality (1.1) for all distinct $x, y \in X$. Note that for all distinct $x, y \in X$ and for $s=\frac{6}{5}$ the inequalities $\frac{5}{12} d(x, T x)<d(x, y)$ and $\alpha(x, y) \geqslant 1$, give

$$
(x, y) \in\{(1,2),(2,1),(2,5),(5,2),(1,5),(5,1),(3,4),(4,3)\} .
$$

So, this implies that

$$
\begin{aligned}
\zeta\left(s^{4} \alpha(x, y) d(T x, T y), M_{T}(x, y)\right) & =\zeta\left(\left(\frac{6}{5}\right)^{4} \alpha(x, y) d(T x, T y), M_{T}(x, y)\right) \\
& =\frac{11}{12} M_{T}(x, y)-\left(\frac{6}{5}\right)^{4} \alpha(x, y) d(T x, T y) \geqslant 0
\end{aligned}
$$

implies that

$$
\left(\frac{6}{5}\right)^{4} \alpha(x, y) d(T x, T y) \leqslant \frac{11}{12} M_{T}(x, y)=\frac{11}{12}\left[\max \left\{d(x, y), d(x, T x), d(y, T y), \frac{d(x, T y)+d(y, T x)}{12 / 5}\right\}\right] .
$$

Now, we consider the following cases.

(i) If $x, y \in\{1,2,5\}$, then

$$
\left(\frac{6}{5}\right)^{4} \alpha(x, y) d(T x, T y)=0 \leqslant \frac{11}{12} M_{T}(x, y)
$$

(ii) If $x=3$ and $y=4$, then

$$
\left(\frac{6}{5}\right)^{4} \alpha(3,4) d(T 3, T 4)=2.11 \leqslant \frac{11}{12} M_{T}(3,4)=2.20 .
$$

That is $\frac{5}{12} d(x, T x)<d(x, y)$ and $\alpha(x, y) \geqslant 1$ implies that $\zeta\left(\left(\frac{6}{5}\right)^{4} \alpha(x, y) d(T x, T y), M_{T}(x, y)\right) \geqslant 0$ for all distinct $x, y \in X$. Moreover, there exists $x_{0} \in X$ such that $\alpha\left(x_{0}, T x_{0}\right) \geqslant 1$. In fact, for $x_{0}=1$, we have $\alpha(1, \mathrm{~T} 1)=\alpha(1,2)=1$. Here all conditions of Theorem 2.1 hold, therefore T has a fixed point. Here, $x=2$ is a fixed point of $T$.

Remark 2.4. In b-metric space defined as above, Theorem 3.4 in [6] fails. By choosing $x=2$ and $y=4$, we have $\zeta\left(\frac{6}{5} d(T 2, T 4), d(2,4)\right)<0$. Thus it is not a b-simulation function.

Corollary 2.5. Let $(\mathrm{X}, \mathrm{d})$ be a complete $\mathrm{b}$-metric space with coefficient $\mathrm{s} \geqslant 1$ and $\alpha: X \times X \rightarrow \mathbb{R}$ be a function. A mapping $\mathrm{T}: \mathrm{X} \rightarrow \mathrm{X}$ be a self mapping and $\zeta \in Z$. Suppose that the following conditions are satisfied:

(i) $T$ is Suzuki type $\alpha-z$ contraction with respect to $\zeta$, i.e.,

$$
\frac{1}{2 s} d(x, T x)<d(x, y) \Rightarrow \zeta\left(s^{4} \alpha(x, y) d(T x, T y), d(x, y)\right) \geqslant 0,
$$

for all distinct $\mathrm{x}, \mathrm{y} \in \mathrm{X}$;

(ii) $\mathrm{T}$ is a triangular $\alpha$-admissible; 
(iii) there exists $x_{0} \in X$ such that $\alpha\left(x_{0}, T x_{0}\right) \geqslant 1$;

(iv) either $T$ is continuous or for any sequence $\left\{x_{n}\right\}$ in $X$ with $\alpha\left(x_{n}, x_{n+1}\right) \geqslant 1$ for all $n \in \mathbb{N}_{0}$ such that $\mathrm{x}_{\mathrm{n}} \rightarrow \mathrm{x} \in \mathrm{X}$ as $\mathrm{n} \rightarrow \infty$, we have $\alpha\left(\mathrm{x}_{\mathrm{n}}, \mathrm{x}\right) \geqslant 1$ for all $\mathrm{n} \in \mathbb{N}_{0}$.

Then $\mathrm{T}$ has a fixed point $x^{*} \in \mathrm{X}$.

By setting $s=1$ in Theorem 2.1, we deduce the following result.

Corollary 2.6. Let $(\mathrm{X}, \mathrm{d})$ be a complete metric space and $\alpha: \mathrm{X} \times \mathrm{X} \rightarrow \mathbb{R}$ be a function. Let $\mathrm{T}: \mathrm{X} \rightarrow \mathrm{X}$ be a self mapping and $\zeta \in Z$. Suppose that the following conditions are satisfied:

(i) T is generalized Suzuki type $\alpha$-Z-contraction with respect to $\zeta$, i.e.,

$$
\frac{1}{2} d(x, T x)<d(x, y) \Rightarrow \zeta(\alpha(x, y) d(T x, T y), M(x, y)) \geqslant 0,
$$

for all distinct $\mathrm{x}, \mathrm{y} \in \mathrm{X}$, where

$$
M(x, y)=\max \left\{d(x, y), d(x, T x), d(y, T y), \frac{d(x, T y)+d(y, T x)}{2}\right\} ;
$$

(ii) T is a triangular $\alpha$-admissible;

(iii) there exists $\mathrm{x}_{0} \in \mathrm{X}$ such that $\alpha\left(\mathrm{x}_{0}, \mathrm{~T} \mathrm{x}_{0}\right) \geqslant 1$;

(iv) either $T$ is continuous or for any sequence $\left\{x_{n}\right\}$ in $X$ with $\alpha\left(x_{n}, x_{n+1}\right) \geqslant 1$ for all $n \in \mathbb{N}_{0}$ such that $\mathrm{x}_{\mathrm{n}} \rightarrow \mathrm{x} \in \mathrm{X}$ as $\mathrm{n} \rightarrow \infty$, we have $\alpha\left(\mathrm{x}_{\mathrm{n}}, \mathrm{x}\right) \geqslant 1$ for all $\mathrm{n} \in \mathbb{N}_{0}$.

Then $\mathrm{T}$ has a fixed point $\mathrm{x}^{*} \in \mathrm{X}$.

Corollary 2.7. Adding condition (A) to the hypotheses of Corollary 2.5 (resp. Corollary 2.6), we obtain that $x^{*}$ is the unique fixed point of $\mathrm{T}$.

\section{Acknowledgment}

Authors are indebted to the referee for his careful reading of the manuscript and valuable suggestions.

\section{References}

[1] H. Aydi, M. Jellali, E. Karapinar, On fixed point results for $\alpha$-implicit contractions in quasi-metric spaces and consequences, Nonlinear Anal. Model. Control, 21 (2016), 40-56. 1

[2] G. V. R. Babu, D. T. Mosissa, Fixed point in b-metric space via simulation function, Novi Sad J. Math., 47 (2017), 133-147. 1

[3] M. Boriceanu, Strict fixed point theorems for multivalued operators in b-metric space, Int. J. Mod. Math., 4 (2009), 285-301. 1, 1.2

[4] S. Czerwik, Contraction mappings in b-metric spaces, Acta Math. Inform. Univ. Ostraviensis, 1 (1993), 5-11. 1, 1.1

[5] S. Czerwik, Nonlinear set-valued contraction mappings in b-metric spaces, Atti Sem. Mat. Fis. Univ. Modena, 46 (1998), 263-276. 1

[6] M. Demma, R. Saadati, P. Vetro, Fixed point results on b-metric space via Picard sequences and b-simulation functions, Iran. J. Math. Sci. Inform., 11 (2016), 123-136. 2.4

[7] E. Karapinar, P. Kumam, P. Salimi, On $\alpha$-廿-Meir-Keeler contractive mappings, Fixed Point Theory Appl., 2013 (2013), 12 pages. 1, 1.4, 1.5

[8] F. Khojasteh, S. Shukla, S. Radenović, A new approach to the study of fixed point theory for simulation functions, Filomat, 29 (2015), 1189-1194. 1, 1.6, 1.7, 1.8

[9] P. Kumam, D. Gopal, L. Budhia, A new fixed point theorem under Suzuki type Z-contraction mappings, J. Math. Anal., 8 (2017), 113-119. 1, 1.9, 1.10, 1.11

[10] B. Mohammadi, S. Rezapour, N. Shahzad, Some results on fixed points of $\alpha-\psi$-Ćirić generalized multifunctions, Fixed Point Theory Appl., 2013 (2013), 10 pages. 1

[11] M. Pacurar, Sequences of almost contractions and fixed points in b-metric spaces, An. Univ. Vest Timi. Ser. Mat.-Inform., 48 (2010), 125-137. 1 
[12] A. Padcharoen, P. Kumam, P. Saipara, P. Chaipunya, Generalized Suzuki type Z-contraction in complete metric spaces, Kragujevac J. Math., 42 (2018), 419-430. 1, 1.12

[13] J. R. Roshan, V. Parvaneh, Z. Kadelberg, Common fixed point theorems for weakly isotone increasing mappings in ordered b-metric spaces, J. Nonlinear Sci. Appl., 7 (2014), 229-245. 1

[14] J. R. Roshan, V. Parvaneh, S. Sedghi, N. Shobkolaei, W. Shatanawi, Common fixed points of almost generalized $(\psi, \phi)$ scontractive mappings in ordered b-metric spaces, Fixed Point Theory Appl., 2013 (2013), 23 pages. 1

[15] B. Samet, C. Vetro, P. Vetro, Fixed point theorem for $\alpha-\psi$-contractive type mappings, Nonlinear Anal., 75 (2012), 2154-2165. 1, 1.3 